\title{
Temperature-controlled metal/ligand stoichiometric ratio in Ag-TCNE coordination networks
}

\author{
Jonathan Rodríguez-Fernández, ${ }^{1}$ Koen Lauwaet, ${ }^{2}$ Maria Ángeles Herranz, ${ }^{3}$ \\ Nazario Martín, ${ }^{2,3}$ José María Gallego, ${ }^{2,4}$ Rodolfo Miranda, ${ }^{1,2}$ and Roberto Otero ${ }^{1,2}$ \\ ${ }^{1}$ Departamento de Física de la Materia Condensada, Universidad Autónoma de Madrid, Cantoblanco, \\ 28049 Madrid, Spain \\ ${ }^{2}$ IMDEA Nanoscience, $c \backslash$ Faraday 9, Campus de Cantoblanco, 28049 Madrid, Spain \\ ${ }^{3}$ Departamento de Química Orgánica, Facultad de Ciencias Químicas, Universidad Complutense de Madrid, \\ Ciudad Universitaria s/n, Madrid, Spain \\ ${ }^{4}$ Instituto de Ciencia de Materiales de Madrid, ICMM, CSIC, Sor Juana Inés de la Cruz $\backslash \backslash n, 28049$ Madrid, \\ Spain
}

(Received 17 November 2014; accepted 10 February 2015; published online 25 February 2015)

\begin{abstract}
The deposition of tetracyanoethylene (TCNE) on $\operatorname{Ag}(111)$, both at Room Temperature (RT, 300 $\mathrm{K})$ and low temperatures $(150 \mathrm{~K})$, leads to the formation of coordination networks involving silver adatoms, as revealed by Variable-Temperature Scanning Tunneling Microscopy. Our results indicate that TCNE molecules etch away material from the step edges and possibly also from the terraces, which facilitates the formation of the observed coordination networks. Moreover, such process is temperature dependent, which allows for different stoichiometric ratios between Ag and TCNE just by adjusting the deposition temperature. X-ray Photoelectron Spectroscopy and Density Functional Theory calculations reveal that charge-transfer from the surface to the molecule and the concomitant geometrical distortions at both sides of the organic/inorganic interface might facilitate the extraction of silver atoms from the step-edges and, thus, its incorporation into the observed TCNE coordination networks. (C) 2015 AIP Publishing LLC. [http://dx.doi.org/10.1063/1.4913326]
\end{abstract}

\section{INTRODUCTION}

The fabrication of $2 \mathrm{D}$ metal-organic coordination networks on solid surfaces has become a very active field of research due to the possible applications of these systems in fields as different as molecular magnetism or heterogeneous catalysis. ${ }^{1-3}$ The functional properties of the resulting coordination network depend on the chemical nature of ligands and metallic centers, the size of the ligands (which determines pore sizes and metal-metal distances), the ligand/metal ratio in those systems in which several stoichiometries are possible, etc. $^{1-3}$

Although the fabrication procedure for such networks usually implies codeposition of organic and inorganic material on the solid surface, it was found that substrate metallic atoms might actually participate in coordination bonds with the organic ligands. ${ }^{4}$ The inclusion of surface atoms in coordination networks with organic ligands has been thoroughly investigated on copper surfaces, where the high reactivity of the spontaneously formed surface adatoms facilitates the formation of metal-organic networks. Similar coordination networks with substrate adatoms have also been observed on other noble metal surfaces, such as $\mathrm{Au}(111),{ }^{5-7} \operatorname{Ag}(110),{ }^{8}$ and $\operatorname{Ag}(111) \cdot{ }^{9,10}$ For silver and gold surfaces, the inclusion of the relatively unreactive surface adatoms into coordination networks is achieved by using very reactive molecules such as surface radicals arising from on-surface dehalogenation of halogenated compounds, ${ }^{8-10}$ or very strong organic acceptors such as fluorinated tetracyanoquinodimethane (F4-TCNQ). ${ }^{7}$
While the participation of adatoms arising from the metal surface facilitates the preparation of coordination networks, it also limits our ability to tune their physico-chemical properties. Of course, using surface atoms as metal centers in coordination networks determines the chemical nature of the metal centers, but moreover the coordination networks with substrate adatoms reported so far in previous studies show a unique value for the metal/ligand stoichiometric ratio. ${ }^{1-10}$ Actually, raising the temperature should enhance the availability of surface adatoms, thereby enabling the formation of coordination networks with different metal/ligand stoichiometries. Different metal/ligand stoichiometries have readily been observed by codepositing $\mathrm{Fe}$ atoms and carboxylic acid molecules in different relative amounts. ${ }^{11}$ The experimental results hitherto reported for coordination of organic ligands with adatoms from the substrate show, however, that only one bonding environment is observed for the metal centers, and this local bonding geometry determines completely the metal/ligand stoichiometric ratio.

In this paper, we show by Variable-Temperature Scanning Tunneling Microscopy (VT-STM) that tetracyanoethylene (TCNE, see inset in Figure 1(a)) molecules deposited on $\mathrm{Ag}(111)$ surfaces at different temperatures form different coordination compounds with substrate $\mathrm{Ag}$ adatoms with varying metal/ligand stoichiometric ratio. This effect occurs even at $150 \mathrm{~K}$, a temperature at which the density of diffusing silver adatoms is negligible. At this temperature, however, the step-edge morphology is observed to be seriously affected by the presence of TCNE molecules, showing deep troughs and 

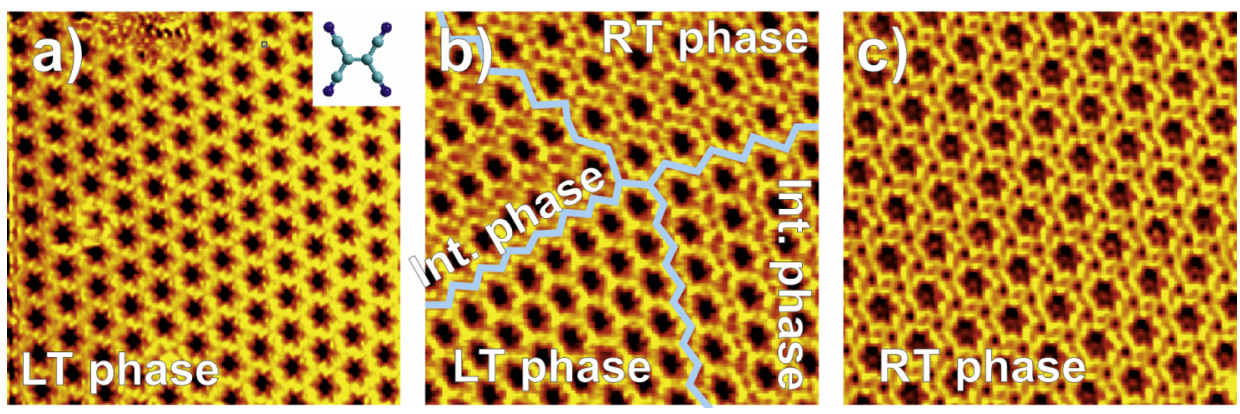

FIG. 1. STM images $(196 \AA \times 196 \AA)$ of the $\operatorname{Ag}(111)$ surface after depositing almost exactly $1 \mathrm{ML}$ of TCNQ at three different temperatures: (a) $150 \mathrm{~K}$; (b) $210 \mathrm{~K}$; and (c) $300 \mathrm{~K}$. The images were taken close to the deposition temperature. (a) $V_{b}=-1.3 \mathrm{~V}, I_{t}=0.27 \mathrm{nA}$; (b) $V_{b}=-2.2 \mathrm{~V}, I_{t}=0.36 \mathrm{nA}$; (c) $V_{b}$ $=-1.5 \mathrm{~V}, I_{t}=0.36 \mathrm{nA}$.

frequent kinks that lead us to hypothesize that the Ag adatoms involved in the formation of the coordination networks have actually been removed from the step edges. Increasing substrate temperature seems to promote the efficiency of this process, leading to coordination networks richer in $\mathrm{Ag}$ adatoms. Comparison with X-ray Photoelectron Spectroscopy results and Density Functional Theory (DFT) calculations allows us to propose a model in which charge-transfer from the silver substrate to the TCNE molecules enables them to form rather strong bonds which facilitate the ejection of silver atoms.

\section{EXPERIMENTAL SECTION}

Film growth and STM and X-ray photoelectron spectroscopy (XPS) investigations were carried out in ultrahighvacuum (UHV) conditions, with a base pressure of $\sim 2$ $\times 10^{-10}$ Torr. Atomically flat, crystalline $\operatorname{Ag}(111)$ surfaces were prepared by standard sputter/anneal procedures (sputter with $1 \mathrm{kV} \mathrm{Ar}^{+}$ions for $10 \mathrm{~min}$ followed by annealing to $800 \mathrm{~K}$ for another $10 \mathrm{~min})$, resulting in large terraces ( $200 \mathrm{~nm}$ wide) separated by monoatomic steps. TCNE was sublimated from a glass crucible held at room temperature onto the clean $\mathrm{Ag}(111)$ substrate, which could be held at any temperature between 150 and $350 \mathrm{~K}$.

The UHV vacuum chamber was equipped with a variable temperature "Aarhus" type STM purchased from SPECS. From the STM chamber, the samples were transferred insitu to the XPS chamber, where photoemission spectra were recorded using Al Ka X-rays of $1486.7 \mathrm{eV}$ with an ellipsoidal monochromator, also from SPECS. STM images were taken both before and after the X-ray measurements, to check for any possible influence of the radiation in the sample structure. Since some radiation damage could be observed after room temperature measurements (see Figure S1 in the supplementary material ${ }^{12}$ ), the XPS spectra shown here were collected with the sample held at $150 \mathrm{~K}$.

\section{RESULTS AND DISCUSSION}

Figure 1 shows typical STM images taken after depositing TCNE on the $\operatorname{Ag}(111)$ surface with the substrate held at (a) $150 \mathrm{~K}$; (b) $210 \mathrm{~K}$; and (c) $300 \mathrm{~K}$. Both at low (LT, Figure 1(a)) and room temperature (RT, Figure 1(c)), TCNE molecules selfassemble into a porous network with hexagonal symmetry, similar to a honeycomb structure but with a different motif and unit cell, as will be discussed later. At the level of resolution of Figure 1, the main difference is that, while the hexagonal pores in the LT phase appear with a single wall between them (Figure 1(a)), those corresponding to the RT phase seems to be separated by double molecular walls (Figure 1(c)). The distance between the pores changes accordingly, going from $18.9 \pm 1 \AA$ for the LT phase to $23.3 \pm 1 \AA$ for the RT phase. The two phases are found to coexist at intermediate deposition temperatures (Figure 1(b)), along with a third intermediate phase characterized by the existence of both single and double walls between the hexagonal pores. The same RT arrangement can be obtained by depositing TCNE at low temperature and then annealing the system to RT. This transition, however, is irreversible: once the room temperature arrangement is obtained, lowering the temperature does not modify the situation, demonstrating that actually the RT phase is lower in energy than the LT phase, but it is unattainable at LT because of kinetic limitations that will become clear later on.

The formation of porous networks on $\mathrm{Ag}(111)$ contrasts with the close-packed assembly that has been reported to result from deposition of TCNE on $\mathrm{Ag}(100) .{ }^{13,14}$ In the last case, the intermolecular interactions are probably substrate mediated due to strong reshaping of the interface that has been reported for $\mathrm{TCNE}^{15}$ and the closely related $\mathrm{TCNQ}^{16}$ on $\mathrm{Cu}(100)$. Substrate mediated interactions can be expected to be rather non-directional and thus lead to close-packed molecular arrays. In our case, however, the porosity of the network indicates that the interactions holding the TCNE molecules together are rather directional, like hydrogen bonds or metal coordination bonds.

Figures 2(a) and 2(d) show high resolution STM images indicating the molecular-scale structure of the LT (a) and RT (d) phases of TCNE on $\mathrm{Ag}(111)$. Each molecule appears as a slightly elongated ellipsoid aligned with the high-symmetry directions of the $\operatorname{Ag}(111)$ surface. The lengths of the sides of the hexagonal unit cell are equal to the previously mentioned interpore distances, which are compatible with commensurate structures described by the epitaxial relationships $\left(\begin{array}{cc}5 & -2 \\ 7 & 5\end{array}\right)$ and $\left(\begin{array}{cc}5 & -5 \\ 10 & 5\end{array}\right)$, respectively. These lattice structures correspond to about $10^{-2}$ molecules $/ \AA^{2}$ or about 1 molecule every 13 substrate atoms for the LT phase, while the RT phase is denser, with $1.1 \times 10^{-2}$ molecules $/ \AA^{2}$ or about 1 molecule every 12.5 substrate atoms.

In order to understand the origin of the intermolecular interactions leading to the stabilization of the previously described networks, we have performed DFT calculations for both freestanding 2D molecular layers and molecular 

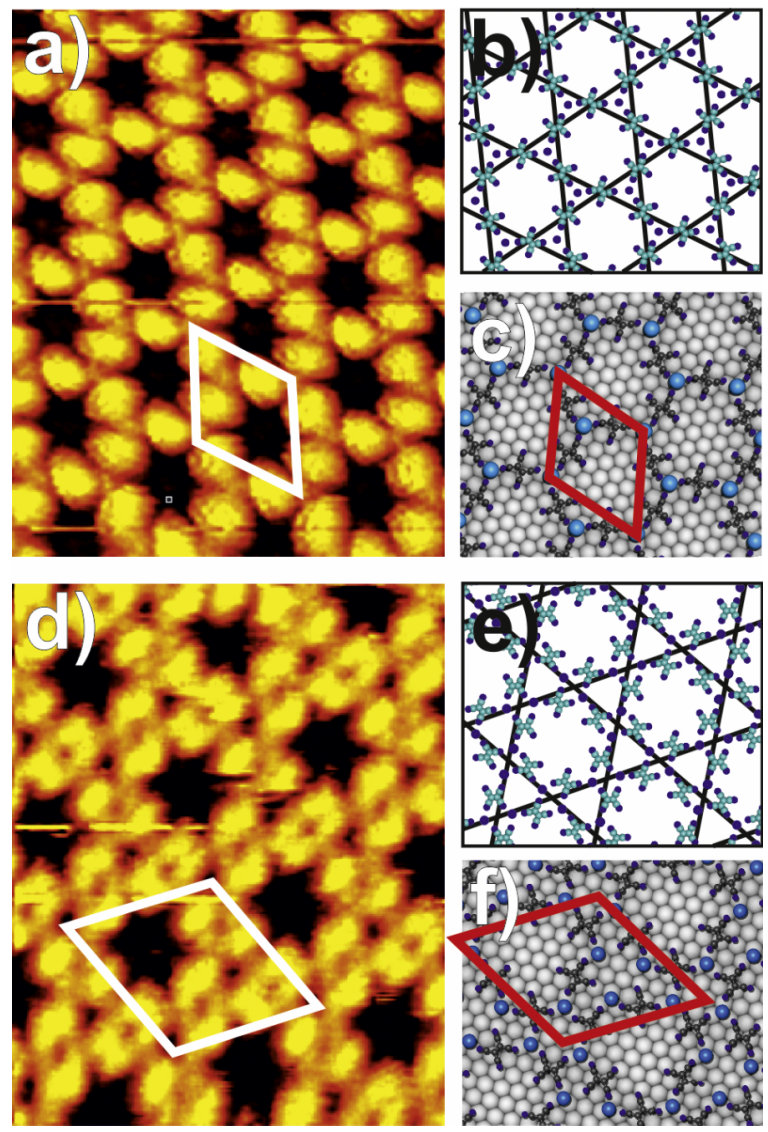

FIG. 2. (a) and (d) Close-up STM images ( $68 \AA \times 86 \AA$ ) of the two different arrangements obtained after depositing TCNE on $\operatorname{Ag}(111)$ at (a) $150 \mathrm{~K}$; and (b) $300 \mathrm{~K}$. (a) $V_{b}=1.3 \mathrm{~V}, I_{t}=0.29 \mathrm{nA}$; (d) $V_{b}=-1.2 \mathrm{~V}, I_{t}=0.19 \mathrm{nA}$. The unit cell is marked by a white rhombus. (b) and (e) Proposed models for the structure of the networks observed after depositing TCNE on $\operatorname{Ag}(111)$ (b) at low temperature, and (e) room temperature in the absence of the substrate. (c) and (f) Results of DFT calculations for the optimized arrangement of two different Ag-TCNE coordination networks including the substrate. In (c) and (f), the silver adatoms are colored in blue, whereas the substrate atoms are colored in gray, and the unit cell is identified by a red rhombus.

arrangements supported by a $\operatorname{Ag}(111)$ surface (see Sec. S2 in the supplementary material ${ }^{12}$ ). Our calculations revealed that the optimized molecular arrangements obtained in the absence and in the presence of the surface are remarkably similar, the role of the solid surface being mostly that of modifying the molecular conformations out of perfect planarity (see discussion below) and of fixing the orientation of the molecular layers with respect to the lattice of surface atoms. The lattice parameters obtained for the LT and RT phases in these calculations are 14.9 and $20.8 \AA$ (see Figure S2(a) and S2(b) in the supplementary material ${ }^{12}$ ), well below the experimental values of $18.9 \pm 1 \AA$ (LT) and $23.3 \pm 1 \AA$ (RT). Notice that increasing the lattice parameters from the theoretically obtained ones to the experimental values would imply to enlarge first neighbors distances by more than $25 \%$ in the LT case and $12 \%$ in the RT case, which for intermolecular distances like the ones we obtain experimentally $(\sim 8 \AA)$ imply distances between 1 and $2 \AA$. These changes in distances are too large not to affect significantly the stabilizing effect of hydrogen bonds or other local electrostatic interactions and suggest that something else must be involved in mediating the intermolecular interactions, keeping them strong at relatively large intermolecular separations.

The previous discussion, along with the realization that the STM images show relatively often the existence of small bright protrusions between the ellipsoidal TCNE molecules (see Figures 2(a) and 2(d)), suggest that silver adatoms might be incorporated in the network by metal coordination. To test this hypothesis, we have repeated the previous calculations for free-standing TCNE layers but now including silver adatoms at the nodes of the networks (Figures 2(b) and 2(e)). Notice that both the RT and LT phases can be considered as examples of Kagome lattices (see Figures 2(b) and 2(e)): while for the LT phase there is quite obviously one molecule at each node of the lattice (Figure 2(b)), the RT phase can be understood as a Kagome lattice with two molecules per node (see Figure 2(e)). For the LT phase, STM images suggest that just one silver adatom needs to be included at the center of each triangle of the Kagome lattice (Figure 2(b)), with a stoichiometry of 2 $\mathrm{Ag}$ adatoms per 3 TCNE molecules (2:3 Ag:TCNE ratio, or 1 $\mathrm{Ag}$ adatom every 19.5 substrate atoms). The RT phase, on the other hand, requires two silver adatoms per TCNE pair (1:1 $\mathrm{Ag}$ :TCNE ratio, or $1 \mathrm{Ag}$ adatom every 12.5 substrate atoms), each of which is also involved in the bonding among three pairs to build the Kagome lattice (Figure 2(e)). The similarity with the experimental results is now quite satisfactory. The lattice parameters for the calculated structures are $18.4 \AA$ (LT) and $23.2 \AA$ (RT), which are very close to the experimental ones (see Figures S2(c) and S2(d)). Moreover, including the substrate in the calculations (Figures 2(c) and 2(f)) only changes slightly the molecular conformations and directions, but the main features of the lattice remain unchanged. We thus conclude that silver adatoms do readily coordinate to TCNE molecules to form ordered structures with a metal/ligand stoichiometry that can be sensitively tuned by changing the substrate's temperature.

The origin of the $\mathrm{Ag}$ adatoms which get incorporated into the TCNE lattice still has to be explained. The activation barrier for atom detachment from a step in $\operatorname{Ag}(111)$ has been estimated to be $0.71 \mathrm{eV},{ }^{17}$ which makes diffusion along step edges the dominant mechanism explaining step fluctuations on this surface, even at room temperature. ${ }^{18,19}$ Because of the high energy cost associated with step-detachment, the equilibrium concentration of $\mathrm{Ag}$ adatoms on the terraces, $\theta_{\mathrm{ad}}=\exp \left(-E_{a} / k T\right)$, where $E_{a}$ is the energy required to bring an atom from a kink onto a terrace, is expected to be very low (of the order of $10^{-12}$ per substrate atom at $300 \mathrm{~K}$ ). This suggests that the abundant ejection of $\mathrm{Ag}$ adatoms is strictly related to the presence of TCNE molecules. This is actually demonstrated by our STM images: step etching processes occur in the presence of TCNE even at relatively low temperatures $(150 \mathrm{~K})$. Figure 3(a) shows a detail of a $\mathrm{Ag}(111)$ step-edge after depositing $0.4 \mathrm{ML}$ of TCNE at $160 \mathrm{~K}$. Contrary to what is observed before deposition (see the inset in Figure 3(a)), the step looks very rough, with clear indications of loss of material. Notice that this removal often takes place by creating rather long (up to $\sim 30 \AA$ ) vacancy chains along the high-symmetry directions of the substrate, which is a rather unusual etching geometry. Notice that the formation of $1 \mathrm{D}$ vacancy chains indicates that the molecules involved in 

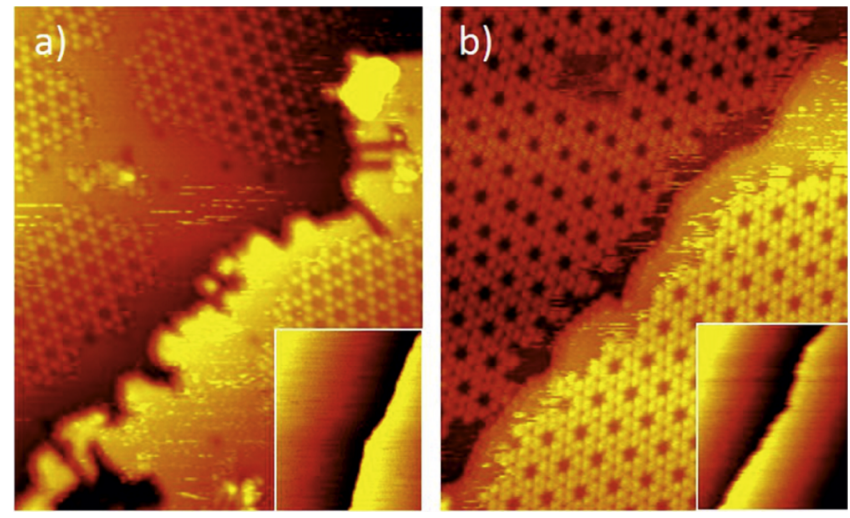

FIG. 3. STM images $(277 \AA \times 344 \AA)$ taken after depositing TCNE on $\mathrm{Ag}(111)$ at (a) $150 \mathrm{~K}$; (b) $300 \mathrm{~K}$. The STM images were taken at the deposition temperature. The insets in (a) and (b) show STM images ( $277 \AA \times 344 \AA$ ) of the clean $\operatorname{Ag}(111)$ surface taken at (a) $130 \mathrm{~K}$; (b) $300 \mathrm{~K}$. (a) $V_{b}=1.74 \mathrm{~V}$, $I_{t}=0.31 \mathrm{nA}$; (b) $V_{b}=-1.74 \mathrm{~V}, I_{t}=0.68 \mathrm{nA}$; inset (a) $V_{b}=-0.42 \mathrm{~V}$, $I_{t}=1.15 \mathrm{nA}$; inset (b) $V_{b}=-2.22 \mathrm{~V}, I_{t}=0.29 \mathrm{nA}$.

step etching are the molecules from the upper terrace. For a molecule from the lower terrace to extract an atom from the end of the 1D chain, it would have to make its way to the end of the chain. Even if the TCNE molecules could actually fit within the $1 \mathrm{D}$ vacancy chain, it would thus become very strongly attached to it. Such strong attachment would hinder the extraction of more material out of the incipient vacancy chain. Of course this hindrance would not be there if the molecule is sitting in the upper terrace. At room temperature, the probability of step etching increases, the population of silver adatoms on the surface increases accordingly, and a new coordination network with a higher Ag:TCNE ratio is formed. At room temperature, however, the steps recover their original shape, thanks to a self-healing process due to the above mentioned atomic diffusion along the steps.

Step etching and reconstruction induced by molecular adsorbates have been previously observed. ${ }^{20-24}$ Very recently, step etching of the $\mathrm{Cu}(100)$ surface after room temperature deposition of F4-TCNQ has also been reported..$^{23} \mathrm{We}$ hypothesize that in our case, the mass transport associated to step etching provides sufficient silver adatoms to fabricate coordination networks even at $150 \mathrm{~K}$.

The process by means of which TCNE adsorption facilitates $\mathrm{Ag}$ adatom ejection is probably related to the strong reshaping of the molecule-metal interface associated to charge transfer between the strong acceptors containing dicyanomethylene end groups and the metal surface, which has also been suggested to play a role in mediating new stressrelated interadsorbate interactions for TCNQ adsorbates ${ }^{16}$ and in the decyanation of relatively larger species of the same family. ${ }^{25}$ Figure 4 shows the $\mathrm{C} 1 \mathrm{~s}$ and N1s core level corresponding to (a) the low temperature arrangement and (b) the room temperature arrangement. The bottom spectra (c) correspond to the clean Ag surface. In both the LT and RT structures, the $\mathrm{C} 1 \mathrm{~s}$ peak appears at $285.4 \mathrm{eV}$ and the N1s peaks at $398.2 \mathrm{eV}$. (The two broad peaks appearing at $\sim 398.5$ and $393.0 \mathrm{eV}$ in the $\mathrm{N} 1 \mathrm{~s}$ spectra of the clean $\mathrm{Ag}$ surface are due to bulk plasmon losses ${ }^{26}$ ). As discussed in Figure S3 in the supplementary material, ${ }^{12}$ the superposition of the XPS peak
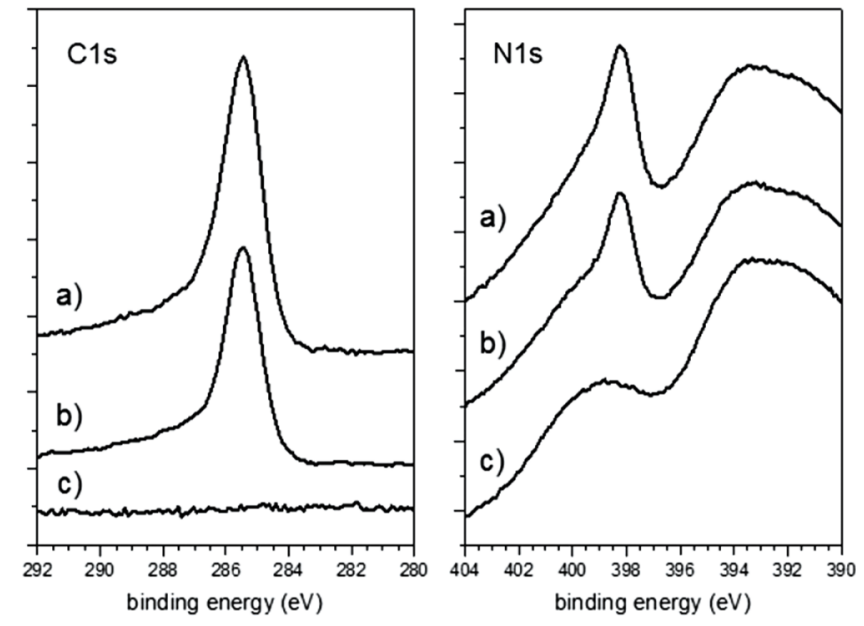

FIG. 4. C1s and N1s regions of the XPS spectra $(h v=1486 \mathrm{eV})$ taken after depositing TCNE on $\operatorname{Ag}(111)$ : (a) at $150 \mathrm{~K}$, and (b) at room temperature. The curve (c) shows the spectra recorded on a clean $\operatorname{Ag}(111)$ surface. All the spectra were measured at $150 \mathrm{~K}$.

to the plasmonic losses does not affect the measured binding energy within experimental error. For TCNE salts, where the TCNE molecules is negatively charged, the $\mathrm{C} 1 \mathrm{~s}$ and $\mathrm{N} 1 \mathrm{~s}$ peaks appear around 285.9 and $398.8 \mathrm{eV}$, respectively, ${ }^{27-29}$ which are closer to the experimentally found binding energies. The similar TCNQ molecule when adsorbed on $\mathrm{Cu}(100)^{16}$ and graphene $/ \mathrm{Ru}(0001)^{30}$ is known to become charged by electron transfer from the surface, and XPS experiments show a N1s core level at 398.7 and $398.3 \mathrm{eV}$, respectively. On the other hand, for TCNQ on graphene/Ir(111) of in multilayers, where no charge transfer takes place, the N1s core level appears at $399.3 \mathrm{eV}^{30}$ This confirms that TCNE is charged upon adsorption on $\operatorname{Ag}(111)$.

Previous studies on the adsorption of molecules from the same family on noble metal surfaces have repeatedly shown that significant structural changes are related to the uptake of electrons by the organic acceptor. ${ }^{13-16}$ To gain insight into this problem, we have performed DFT calculations of a TCNE molecule on the $\operatorname{Ag}(111)$ surface. Of all the tested possibilities, the minimum energy configuration is shown in Figure 5. In good agreement with our XPS results, the calculations indicate that the molecule accepts $\sim 0.88 \mathrm{e}^{-}$from the silver substrate. Moreover, when the TCNE molecule is coordinated to the silver adatoms (see Figures 3(a) and 3(b)), the total charge on the TCNE molecule is approximately the same $\left(\sim 0.86 \mathrm{e}^{-}\right)$ according to DFT calculations for the LT network. That is, a strong electron acceptor, such as TCNE, on the silver surface gets negatively charged, irrespectively of the coordination with silver adatoms. In the absence of adatoms, the molecule takes all the charge from the silver substrate. If the coordination network exists, TCNE takes part of the electron from the substrate and part from the silver adatom (the calculated charge on the silver adatom is $0.45 \mathrm{e}^{-}$).

From the structural point of view, the TCNE molecule is slightly bent, with the $\mathrm{N}$ atoms closer to the silver surface (by $0.30 \AA$ ) than the central $\mathrm{C}$ atoms. Importantly, the $\mathrm{Ag}$ atoms directly below the $\mathrm{N}$ atoms are slightly pulled up, $0.29 \AA$ higher than the ideal surface termination. This situation is 

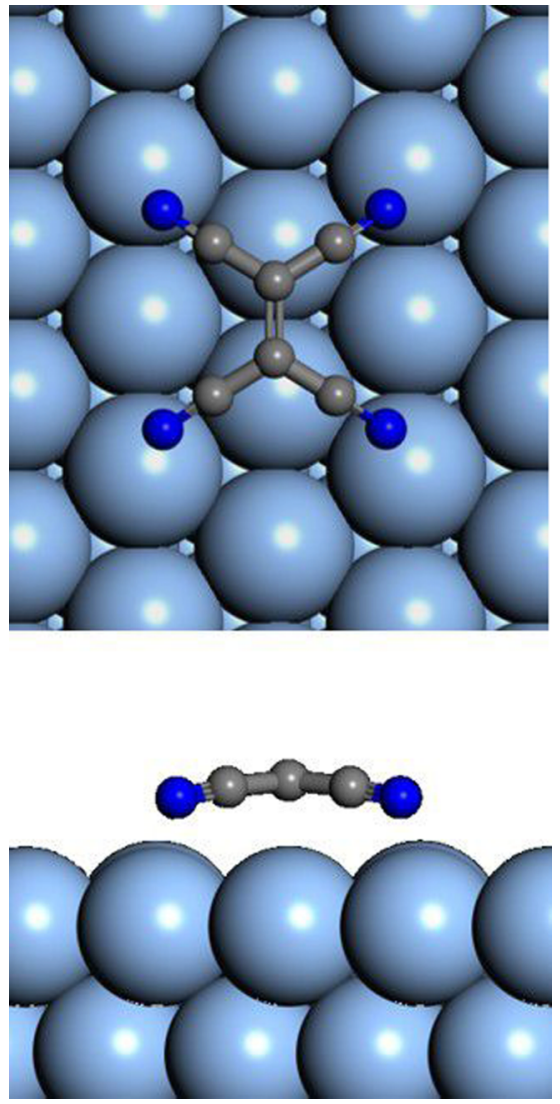

FIG. 5. Results of DFT calculations showing the optimized conformation of an isolated TCNE molecule on the $\mathrm{Ag}(111)$ surface.

very similar to that observed when TCNE is adsorbed on $\mathrm{Cu}(100)^{15}$ or TCNQ on $\mathrm{Cu}(100) .{ }^{16}$ In there, the acceptor molecules strongly modify the copper surface, in such a way that the highest copper atoms act as bridges between the TCNE molecules. Notice that pulling the $\mathrm{Ag}$ atoms out of their lattice equilibrium positions might be considered as an early stage of the ejection of the $\mathrm{Ag}$ adatoms. Actually, given the low coordination of atoms at step edges, a TCNE molecule at the upper terrace might easily extract the atom and take it with itself to the upper terrace, or just facilitate the ejection to the lower terrace.

\section{CONCLUSIONS}

We have shown that, upon deposition of TCNE on $\operatorname{Ag}(111)$ at 150 and $300 \mathrm{~K}$ two different Ag-TCNE coordination networks, with different stoichiometries are formed. We have actually demonstrated that the silver adatoms arise at least partially from the molecule induced step etching of the silver step edges. This result lifts the condition that the density of diffusing adatoms be high on metal substrates for them to get incorporated into coordination networks: if the organic ligands are chosen properly, they can actually extract the atoms from step-edges. While most of the results on coordination networks where the metal center is a substrate atom has so far been carried out using noble metal surfaces, with low cohesive energies and, thus, a high density of free surface adatoms, it should now be possible to use a larger variety of transition metal surfaces. It is worthy to note that if deposition were done at room temperature, the molecule induced step etching would be hard to guess, since at this temperature the steps practically recover the equilibrium room temperature shape. This result is also important because of the small size of TCNE. TCNE is one of the smallest molecules involved in metal organic coordination networks (together with the carboxylic acid $\left.\mathrm{C}_{2} \mathrm{O}_{4} \mathrm{H}_{2}\right),{ }^{31,32}$ and therefore able to provide a rather short metal-to-metal distance. This is probably involved in the magnetic properties that have been reported for TCNE metal-coordination networks, with metalmetal distances between 7 and $10 \AA .{ }^{33,34}$ However, to our knowledge, no extended purely 2D network has been reported using TCNE, and only the bottom-up fabrication by atomic manipulation of small V-TCNE clusters on $\mathrm{Ag}(100)$ has been reported. ${ }^{35}$ Our results suggest that during the growth of TCNE coordination material by Chemical Vapor Deposition methods for organic magnetic applications, possible substitution of the magnetic metal centers by non-magnetic substrate adatoms might lead to weaker magnetic responses.

\section{ACKNOWLEDGMENTS}

This work has been financed by the Spanish MICINN (Project Nos. FIS2010-18847, FIS2012-33011, CTQ201017006, CTQ2011-24652, Project CONSOLIDER-INGENIO EN NANOCIENCIA MOLECULAR Ref. No. CSD200700010), and the Comunidad de Madrid (Project Nos. S2009/MAT1726 and FotoCarbon-S2013/MIT-2841).

${ }^{1}$ J. V. Barth, Surf. Sci. 603, 1533 (2009).

${ }^{2}$ N. Lin, S. Stepanow, M. Ruben, and J. V. Barth, Top. Curr. Chem. 287, 1 (2009).

${ }^{3}$ J. V. Barth, Annu. Rev. Phys. Chem. 58, 375 (2007)

${ }^{4}$ N. Lin, A. Dmitriev, J. Weckesser, J. V. Barth, and K. Kern, Angew. Chem., Int. Ed. 41, 4779 (2002).

${ }^{5}$ P. Maksymovych, D. C. Sorescu, and J. T. Yates, Phys. Rev. Lett. 97, 146103 (2006).

${ }^{6}$ Z. Shi and N. Lin, J. Am. Chem. Soc. 131, 5376 (2009).

${ }^{7}$ M. N. Faraggi, N. Jiang, N. González-Lazunka, A. Langner, S. Stepanow, K. Kern, and A. Arnau, J. Phys. Chem. C 116, 24558 (2012).

${ }^{8}$ H. Walch, R. Gutzler, T. Sirtl, G. Eder, and M. Lackinger, J. Phys. Chem. C 114, 12604 (2010).

${ }^{9}$ J. Park, K. Y. Kim, K.-H. Chung, J. K. Yoon, H. Kim, S. Han, and S.-J. Kahng, J. Phys. Chem. C 115, 14834 (2011).

${ }^{10}$ K.-H. Chung, B.-G. Koo, H. Kim, J. K. Yoon, J.-H. Kim, Y.-K. Kwon, and S.-J. Kahng, Phys. Chem. Chem. Phys. 14, 7304 (2012).

${ }^{11}$ S. Stepanow, M. Ligenfelder, A. Dmitriev, H. Spillmann, E. Delvigne, N. Lin, X. Deng, C. Cai, J. V. Barth, and K. Kern, Nat. Mater. 3, 229 (2004).

${ }^{12}$ See supplementary material at http://dx.doi.org/10.1063/1.4913326 for STM images showing the modifications effected by expossure to XPS (Sec. S1), the results of DFT calculations of freestanding layers with and without coordinated $\mathrm{Ag}$ adatoms (Sec. S2) and the effect of normalizing the N1s XPS spectra to the background, with a discussion on the effect of the peak position (Sec. S3).

${ }^{13}$ D. Wegner, R. Yamachika, Y. Wang, V. W. Brar, B. M. Bartlett, J. R. Long, and M. F. Crommie, Nano Lett. 8, 131 (2008).

${ }^{14}$ D. Wegner, R. Yamachika, X. Zhang, Y. Wang, M. F. Crommie, and N. Lorente, Nano Lett. 13, 2346 (2013).

${ }^{15}$ S. Bedwani, D. Wegner, M. F. Crommie, and M. F. Rochefort, Phys. Rev. Lett. 101, 216105 (2008).

${ }^{16}$ T. Tseng, C. Urban, Y. Wang, R. Otero, S. L. Tait, M. Alcamí, D. Écija, M. Trelka, J. M. Gallego, N. Lin, M. Konuma, U. Starke, A. Nefedov, A. Langner, C. Wöll, M. A. Herranz, F. Martín, N. Martín, K. Kern, and R. Miranda, Nat. Chem. 2, 374 (2010).

${ }^{17}$ K. Morgenstern, G. Rosenfeld, E. Laegsgaard, F. Besenbacher, and G. Comsa, Phys. Rev. Lett. 80, 556 (1998). 
${ }^{18}$ M. Poengsen, J. F. Wolf, J. Frohn, M. Giesen, and H. Ibach, Surf. Sci. 274, 430 (1992).

${ }^{19}$ D. B. Dougherty, O. Bondarchuk, M. Degawa, and E. D. Williams, Surf. Sci. 527, L213 (2003).

${ }^{20}$ F. Leibsle, S. Haq, B. G. Frederick, M. Bowker, and N. V. Richardson, Surf. Sci. 343, L1175 (1995).

${ }^{21}$ Q. Chen and N. V. Richardson, Prog. Surf. Sci. 73, 59 (2003).

${ }^{22}$ F. Rosei, M. Schunack, P. Jiang, A. Gourdon, E. Lægsgaard, I. Stensgaard, C. Joachim, and F. Besenbacher, Science 296, 328 (2002).

${ }^{23}$ R. Otero, F. Rosei, Y. Naitoh, P. Jiang, P. Thostrup, A. Gourdon, E. Laegsgaard, I. Stensgaard, C. Joachim, and F. Besenbacher, Nano Lett. 4, 75 (2004).

${ }^{24}$ T. Katayama, K. Mukai, S. Yoshimoto, and J. Yoshinobu, Phys. Rev. B 83, 153403 (2011).

${ }^{25}$ C. Urban, Y. Wang, J. Rodríguez-Fernández, R. García, M. A. Herranz, M. Alcamí, N. Martín, F. Martín, J. M. Gallego, R. Miranda, and R. Otero, Chem. Commun. 50, 833 (2014).

${ }^{26}$ J. Leiro, E. Minni, and E. Suoninen, J. Phys. F: Met. Phys. 13, 215 (1983).
${ }^{27}$ P. Bhatt and S. M. Yusuf, Surf. Sci. 605, 1861 (2011).

${ }^{28}$ E. Carlegrim, Y. Zhan, F. Li, X. Liu, and M. Fahlman, Org. Electron. 11, 1020 (2010)

${ }^{29}$ C. Tengstedt, M. Unge, M. P. de Jong, S. Stafström, W. R. Salaneck, and M. Fahlman, Phys. Rev. B 69, 165208 (2004).

${ }^{30}$ D. Maccariello, M. Garnica, M. A. Niño, C. Navío, P. Perna, S. Barja, A. L. Vázquez de Parga, and R. Miranda, Chem. Mater. 26, 2883 (2014).

${ }^{31}$ M. N. Faraggi, C. Rogero, A. Arnau, M. Trelka, D. Écija, C. Isvoranu, J. Schnadt, C. Marti-Gastaldo, E. Coronado, J. M. Gallego, R. Otero, and R. Miranda, J. Phys. Chem. C 115, 21177 (2011).

${ }^{32}$ R. Otero, J. M. Gallego, A. L. Vázquez de Parga, N. Martín, and R. Miranda, Adv. Mater. 23, 5148 (2011).

${ }^{33}$ K. I. Pokhodnya, A. J. Epstein, and J. S. Miller, Adv. Mater. 12, 410 (2000).

${ }^{34}$ H. Casellas, D. de Caro, L. Valade, and P. Cassoux, Chem. Vap. Deposition 8, 145 (2002).

${ }^{35}$ D. Wegner, R. Yamachika, X. Zhang, Y. Wang, T. Baruah, M. R. Pederson, B. M. Bartlett, J. R. Long, and M. F. Crommie, Phys. Rev. Lett. 103, 087205 (2009). 